\title{
ON BOUNDARY VALUES OF A SCHLICHT MAPPING
}

\author{
ROGER A. HORN ${ }^{1}$
}

1. Introduction. Let $(a, b)$ be a finite interval on the real line, let $C_{0}^{n}(a, b)$ denote all $n$-times continuously differentiable real-valued functions with compact support contained in $(a, b)$ (with $C_{0} \equiv C_{0}^{0}$ ), and let $L_{0}(a, b) \equiv\left\{f \in C_{0}(a, b) \mid \int_{a}^{b} f(x) d x=0\right\}$. It is the aim of this note to provide a simple, purely function-theoretic proof of the following

ThEOREM. Let $f(x)$ be a $C^{1}$ real-valued function with strictly positive derivative on $(a, b)$. The function $f(x)$ is the restriction to $(a, b)$ of $a$ univalent analytic function $f(z)$ mapping the upper half plane (UHP) into itself if and only if

$$
\int_{a}^{b} \int_{a}^{b} h(x) \log \left(\frac{f(x)-f(y)}{x-y}\right) h(y) d x d y \geqq 0 \text { for every } h \in L_{0}(a, b) .
$$

The difference quotient is of course taken to mean $f^{\prime}(x)$ when $y=x$.

FitzGerald [2] recently provided the first proof of the sufficiency of condition (1) by extending Mercer's theorem on the eigenfunction expansion of a nonnegative symmetric kernel, while Loewner's two papers [3] and [4] together show its necessity using his theory of the generation of univalent mappings by the composition of infinitesimal transformations. We shall show that necessity is an immediate consequence of the classical Grunsky inequalities for univalent mappings, and that sufficiency may be deduced from an elementary inequality between a nonnegative Hermitian quadratic form and its associated bilinear form.

2. Lemmata, notation, and a reduction of the theorem. Let $K(x, y)$ be a $C^{2}$ real-valued symmetric kernel. We shall write " $K(x, y) \succeq 0$ on F" whenever the quadratic form

$$
\iint h(x) K(x, y) h(y) d x d y \geqq 0
$$

for every function $h(x)$ in the class $\mathcal{F}$.

LEMMA 1. $K(x, y) \succeq 0$ on $L_{0}(a, b)$ if and only if $\left(\partial^{2} / \partial x \partial y\right) K(x, y) \succeq 0$ on $C_{0}(a, b)$.

Received by the editors June 27, 1966.

1 This work was supported by an NSF Co-operative Fellowship and NSF Grant GP 5358 at Stanford University. 
Proof. If we set $H(x) \equiv \int_{a}^{x} h(t) d t$ whenever $h \in L_{0}(a, b)$, or if we set $h(x) \equiv(d / d x) H(x)$ whenever $H \in C_{0}^{1}(a, b)$, we have in either case both $h \in L_{0}(a, b)$ and $H \in C_{0}^{1}(a, b)$. Integration by parts twice shows that in both cases,

$$
\int_{a}^{b} \int_{a}^{b} h(x) K(x, y) h(y) d x d y=\int_{a}^{b} \int_{a}^{b} H(x) \frac{\partial^{2}}{\partial x \partial y} K(x, y) H(y) d x d y .
$$

Since every function in $C_{0}(a, b)$ may be approximated uniformly by functions of $C_{0}^{\mathbf{1}}(a, b)$, the proof is complete.

Lemma 2. Let $K(x, y) \succeq 0$ on $C_{0}(a, b)$ and let $h(x)$ be any continuous complex-valued function on $[a, b]$. Then

$$
\left|\int_{a}^{b} \int_{a}^{b} h(x) K(x, y) h(y) d x d y\right| \leqq \int_{a}^{b} \int_{a}^{b} h(x) K(x, y) \bar{h}(y) d x d y .
$$

Proof. Since we may approximate $h(x)$ in absolute mean by compactly supported functions, we may assume that $h(x)=u(x)+i v(x)$, where $u, v \in C_{0}(a, b)$. But (2) is then equivalent to

$$
\begin{aligned}
& \left(\iint u(x) K(x, y) v(y) d x d y\right)^{2} \\
& \quad \leqq\left(\iint u(x) K(x, y) u(y) d x d y\right)\left(\iint v(x) K(x, y) v(y) d x d y\right),
\end{aligned}
$$

and if we compute the necessarily nonpositive discriminant of the quadratic inequality

$$
\iint[u(x)+t v(x)] K(x, y)[u(y)+t v(y)] d x d y \geqq 0,
$$

which holds for each real $t$, (3) follows immediately.

For convenience in our discussion of the theorem, we shall normalize by assuming $(a, b)=(-1,1)$, map the open unit disc (UD) in to the finite plane with real slits along $(-\infty,-1]$ and $[1, \infty)$ by the transformation $z \rightarrow 2 z /\left(1+z^{2}\right)$, set $f\left(2 x /\left(1+x^{2}\right)\right) \equiv g(x)$, and notice that condition (1) is then equivalent to the following:

$$
\int_{-1}^{1} \int_{-1}^{1} h(x) \log \left(\frac{g(x)-g(y)}{(x-y)(1-x y)}\right) h(y) d x d y \geqq 0
$$

$$
\text { for every } h(x) \in L_{0}(-1,1) \text {. }
$$

Define 


$$
G(x, y) \equiv \frac{\partial^{2}}{\partial x \partial y} \log \left(\frac{g(x)-g(y)}{(x-y)(1-x y)}\right)=U(x, y)+K(x, y),
$$

where

and

$$
U(x, y) \equiv \frac{g^{\prime}(x) g^{\prime}(y)}{[g(x)-g(y)]^{2}}-\frac{1}{(x-y)^{2}}
$$

$$
K(x, y) \equiv \frac{1}{(1-x y)^{2}} .
$$

Let $\mathrm{UD}^{+} \equiv\{z \in \mathrm{UD} \mid \operatorname{Im}(z)>0\}$ and $\mathrm{UD}^{-} \equiv-\mathrm{UD}^{+}$.

Notice that $g \in C^{3}$ (see $\$ 4$ ) so that

$$
U(x, x)=\frac{1}{6}\left\{\frac{g^{\prime \prime \prime}(x)}{g^{\prime}(x)}-\frac{3}{2}\left(\frac{g^{\prime \prime}(x)}{g^{\prime}(x)}\right)^{2}\right\}
$$

is essentially the Schwarz differential expression for $g(x)$, and, as $g^{\prime}(x)>0, U(x, y)$ is a nonsingular kernel. Finally, using Lemma 1, we see that the Loewner-FitzGerald theorem is equivalent to the following

THEOREM. Let $g(x)$ be a $C^{1}$ real-valued function with strictly positive derivative on $(-1,1)$. The function $g(x)$ is the restriction to $(-1,1)$ of a univalent analytic function $g(z): \mathrm{UD}^{+} \rightarrow \mathrm{UHP}$ if and only if

$$
G(x, y) \succeq 0 \quad \text { on } \quad C_{0}(-1,1) .
$$

3. Necessity of the condition $\left(1^{\prime \prime}\right)$. If we suppose that $g(z)$ is analytic and univalent in the UD, then the Grunsky inequalities must be satisfied. They may be stated (see [1, p. 239], or [6, p. 161]) in the form

$$
\left|\sum_{i, j=1}^{N} U\left(z_{i}, z_{j}\right) \xi_{i} \xi_{j}\right| \leqq \sum_{i, j=1}^{N} K\left(z_{i}, \bar{z}_{j}\right) \xi_{i} \bar{\xi}_{j}
$$

which must hold for each integer $N$, all $\left\{z_{i}\right\}_{i=1}^{N} \subset \mathrm{UD}$, and all $\left\{\xi_{i}\right\}_{i=1}^{N} \subset \boldsymbol{C}$. If, however, we choose real $\left\{\xi_{i}\right\}$ and real $\left.\left\{z_{i}\right\} \subset(-1,1)\right)$ we find that

$$
\sum_{i, j=1}^{N}\left[K\left(x_{i}, x_{j}\right) \pm U\left(x_{i}, x_{j}\right)\right] \xi_{i} \xi_{j} \geqq 0,
$$

so, by a limiting process, we may conclude that

$$
K(x, y) \pm U(x, y) \succeq 0 \text { on } C_{0}(-1,1) .
$$

In particular, we have condition $\left(1^{\prime \prime}\right)$. 
4. Sufficiency of the condition $\left(1^{\prime \prime}\right)$. Following Loewner $[4$, Lemma 5], we see that $\log [(f(x)-f(y)) /(x-y)] \succeq_{0}$ on $L_{0}(a, b)$ implies, using the Schur lemma on "Schur products" of positive semidefinite matrices, that $[(f(x)-f(y)) /(x-y)] \succeq_{0}$ on $C_{0}(a, b)$. Thus (Loewner [5, p. 215]), $f(x)$ is actually real-analytic on $(a, b)$ and possesses a regular analytic continuation onto the finite plane with real slits $(-\infty, a]$ and $[b, \infty)$. Furthermore, $f: \mathrm{UHP} \rightarrow \mathrm{UHP}$, and since $f$ is real on $(a, b)$, Schwarz reflection shows that $f$ maps the lower half plane (LHP) into itself.

Thus condition $\left(1^{\prime \prime}\right)$ guarantees that $g(z)$ has the extension and mapping properties required in the theorem except possibly for univalence. It is clear from their definitions that $U(z, \zeta)$ and $G(z, \zeta)$ are regular in the UD if and only if $g(z)$ is univalent, so we need prove only this regularity.

Because $g: \mathrm{UD}^{+} \rightarrow \mathrm{UHP}, g: \mathrm{UD}^{-} \rightarrow \mathrm{LHP}, g$ is real on $(-1,1)$, and $g^{\prime}(0)>0$, it is clear that $G(z, \bar{\zeta})$ is a regular $z$-analytic, $\zeta$-anti-analytic function on $\mathrm{UD}^{+}$, that $G(z, \bar{\zeta})=G(z, \zeta)$ is real for $z, \zeta \in(-1,1)$, and that $G(z, \zeta)$ is regular analy tic in a full neighborhood of zero. If $0<r<1$, let

$$
\begin{aligned}
I_{r} & \equiv(-r, r), \\
D_{r} & \equiv\{z|| z \mid<r \text { and } \operatorname{Im}(z)>0\} \\
S_{r} & \equiv\{z|| z \mid=r \text { and } \operatorname{Im}(z) \geqq 0\} \\
\mathcal{E}_{r} & \equiv\left\{\Gamma \mid \Gamma \text { is a smooth arc in } D_{r} \text { with endpoints } \pm r\right\} \\
r_{0} & \equiv \sup \left\{r \mid G(z, \zeta) \text { is regular in } D_{r}\right\} .
\end{aligned}
$$

Then $G(z, \zeta)$ has a singularity on $S_{r_{0}}$. We know that $0<r_{0} \leqq 1$ and must prove that $r_{0}=1$.

Suppose $r_{0}<1$, let $r<r_{0}$ and let $h(z)$ be any function regular analytic in an open set containing $I_{r_{0}} \cup D_{r_{0}}$. If $\Gamma \in \mathcal{E}_{r}$, then the Cauchy integral theorem allows us to write

$$
\iint_{I_{\mathrm{r} \times I_{r}}} h(x) G(x, y) h(y) d x d y=\iint_{\Gamma_{\times} \times \Gamma} h(z) G(z, \zeta) h(\zeta) d z d \zeta
$$

and

$$
\iint_{I_{r} \times I_{r}} h(x) G(x, y) \bar{h}(y) d x d y=\iint_{\Gamma \times \Gamma} h(z) G(z, \bar{\zeta}) \bar{h}(\zeta) d z d \bar{\zeta} .
$$

Applying Lemma 2 to the left sides of these identities, we conclude that for analytic $h(z)$ and $\Gamma \in \mathcal{E}_{r}$, 


$$
\left|\iint_{\Gamma \times \Gamma} h(z) G(z, \zeta) h(\zeta) d z d \zeta\right| \leqq \iint_{\Gamma \times \Gamma} h(z) G(z, \bar{\zeta}) \bar{h}(\zeta) d z d \bar{\zeta} .
$$

Now, $G(z, \zeta)$ can have only two types of singularities on $S_{r_{0}}$ : (a) for some $z_{1} \in S_{r_{0}}, g^{\prime}\left(z_{1}\right)=0$, i.e., $G\left(z_{1}, z_{1}\right)=\infty$, or (b) for some $z_{1} \neq z_{2}, z_{i} \in S_{r_{0}}$, $g\left(z_{1}\right)=g\left(z_{2}\right)$, i.e., $G\left(z_{1}, z_{2}\right)=\infty$. We use (4) to exclude each type in turn with the help of an argument similar to that of FitzGerald [2].

If (a) were the case, choose some point $z^{\prime} \in D_{r_{0}}$ near $z_{1} \in S_{r_{0}}$ such that $\left|G\left(z^{\prime}, z^{\prime}\right)\right| \gg \sup _{1 \in D_{r 0}} G(z, \bar{z})$. Let $\Gamma \in \mathcal{E}_{r}$ pass through $z^{\prime}$, where $r_{0}>r>\left|z^{\prime}\right|$. Since any continuous function on $\Gamma$ may be approximated uniformly on $\Gamma$ by polynomials in $z$ [7, p. 39], we may choose as an approximate identity on $\Gamma$ based at $z^{\prime}$ a sequence of polynomials in $z,\left\{h_{n}(z)\right\}$. Then, as $n \rightarrow \infty$,

and

$$
\iint_{\mathrm{\Gamma} \times \Gamma} h_{n}(z) G(z, \zeta) h_{n}(\zeta) d z d \zeta \rightarrow G\left(z^{\prime}, z^{\prime}\right)
$$

$$
\iint_{\Gamma \times \Gamma} h_{n}(z) G(z, \bar{\zeta}) \bar{h}_{n}(\zeta) d z d \bar{\zeta} \rightarrow G\left(z^{\prime}, \bar{z}^{\prime}\right)
$$

Using (4), we arrive at

$$
\left|G\left(z^{\prime}, z^{\prime}\right)\right| \leqq G\left(z^{\prime}, \bar{z}^{\prime}\right) .
$$

Thus, there are no singularities of type (a).

If (b) were the case, choose two points $z^{\prime}, z^{\prime \prime} \in D_{r_{0}}$ near $z_{1}, z_{2} \in S_{r_{0}}$ respectively for which $\left|G\left(z^{\prime}, z^{\prime \prime}\right)\right| \gg \sup _{\zeta, z \in D_{r_{0}}} 3|G(z, \bar{\zeta})| \equiv M$, choose $\Gamma \in \mathcal{E}$, passing through $z^{\prime}$ and $z^{\prime \prime}$, where $r_{0}>r^{\prime}>\max \left(\left|z^{\prime}\right|,\left|z^{\prime \prime}\right|\right)$, and choose two sequences $\left\{k_{n}(z)\right\},\left\{p_{n}(z)\right\}$ of polynomials as approximate identities on $\Gamma$ based at $z^{\prime}$ and $z^{\prime \prime}$ respectively. Let $h_{n}(z)=k_{n}(z)+p_{n}(z)$ and repeat the above limiting process to conclude that

$$
\begin{aligned}
& \left|G\left(z^{\prime}, z^{\prime}\right)+2 G\left(z^{\prime}, z^{\prime \prime}\right)+G\left(z^{\prime \prime}, z^{\prime \prime}\right)\right| \\
& \quad \leqq G\left(z^{\prime}, \bar{z}^{\prime}\right)+2 \operatorname{Re} G\left(z^{\prime}, \bar{z}^{\prime \prime}\right)+G\left(z^{\prime \prime}, \bar{z}^{\prime \prime}\right)
\end{aligned}
$$

or, using (5),

$$
\left|G\left(z^{\prime}, z^{\prime \prime}\right)\right| \leqq G\left(z^{\prime}, \bar{z}^{\prime}\right)+\left|G\left(z^{\prime}, \bar{z}^{\prime \prime}\right)\right|+G\left(z^{\prime \prime}, \bar{z}^{\prime \prime}\right) \leqq M .
$$

Thus, there are no singularities of type (b) either, and the proof is concluded.

5. Acknowledgements. The author is indebted to Mr. C. FitzGerald for giving him access to his unpublished work, to Professor C. Loewner and Professor D. C. Spencer for their help and encouragement, and to Mr. F. Johnson for several fruitful conversations. 


\section{BiBLIOGRAPHY}

1. S. Bergman and M. Schiffer, Kernel functions and conformal mapping, Compositio Math. 8 (1951), 205-249.

2. C. FitzGerald, On analytic continuation to a schlicht function, Proc. Amer. Math. Soc. (to appear).

3. C. Loewner, Semigroups of conformal mappings, Seminars on Analytic Functions, Vol. 1, Institute for Advanced Study, Princeton, N. J., 1957, 278-288.

4. - On schlicht-monotonic functions of higher order, J. Math. Anal. Appl. 14 (1966), 320-325.

5. K. Löwner (C. Loewner), Über monotone Matrixfunktionen, Math Z. 38 (1934), 177-216.

6. M. Schiffer and D. C. Spencer, Functionals of finite Riemann surfaces, Princeton Univ. Press, Princeton, N. J., 1954.

7. J. L. Walsh, Interpolation and approximation by rational functions in the complex domain, 4th rev. ed., Colloq. Publ., Vol. 20, Amer. Math. Soc., Providence, R. I., 1966.

STANFORD UNIVERSITY 